\title{
Quantum Phase Transitions in the One-Dimensional $S=1$ Spin-Orbital Model: Implications for Cubic Vanadates
}

\author{
Satoshi Miyashita, Akira Kawaguchi $⿴ 囗 6$ and Norio Kawakami \\ Department of Applied Physics, Osaka University, Suita, Osaka 565-0871, Japan \\ Giniyat Khaliullin \\ Max-Planck-Institut für Festkörperforschung, Heisenbergstrasse 1, D-70569 Stuttgart, Germany
}

(Dated: October 31, 2018)

\begin{abstract}
We investigate ground-state properties and quantum phase transitions in the one-dimensional $S=1$ spin-orbital model relevant to cubic vanadates. Using the density matrix renormalization group, we compute the ground-state energy, the magnetization and the correlation functions for different values of the Hund's coupling $J_{H}$ and the external magnetic field. It is found that the magnetization jumps at a certain critical field, which is a hallmark of the field-induced first-order phase transition. The phase transition driven by $J_{H}$ is also of first order. We also consider how the lattice-induced ferro-type interaction between orbitals modifies the phase diagram, and discuss the results in a context of the first-order phase transition observed in $\mathrm{YVO}_{3}$ at $77 \mathrm{~K}$.

PACS numbers: 75.30.Et, 73.43.Nq, 75.10.Pq, 71.27.+a
\end{abstract}

\section{INTRODUCTION}

Orbital fluctuations in correlated electron systems have attracted much interest recently. They play a particularly important role in transition metal oxides, where the low-energy physics is controlled by the orbital as well as spin degrees of freedom. Orbitals of $d$-electrons possess characteristic spatial anisotropy, which sometimes results in remarkable phenomena such as the reduction of effective dimensions. For instance, in cubic vanadates such as $\mathrm{YVO}_{3}$ and $\mathrm{LaVO}_{3}$, an effective one-dimensional (1D) system is formed along the $c$-axis at low temperatures, which has been shown by a number of experiments 1.2.3.4.5.6.7 on spin and orbital orderings. For $\mathrm{YVO}_{3}$, there are two magnetic phases: $C$-type antiferromagnetic order (ferromagnetic along $c$-axis and antiferromagnetic in $a b$ plane) at higher temperatures $77 K<T<114 K$, and $G$-type spin order (antiferromagnetic in all three directions) at lower temperatures $T<77 K \stackrel{5}{\underline{5}}$ As for the orbital sector, the $G$-type orbital-antiferromagnetic correlations develop below $T \sim 200 K$ and it changes to the $C$-type order (in-phase alignment of orbitals along the $c$-axis) below $T=77 \mathrm{~K}$. On the other hand, in $\mathrm{LaVO}_{3}$, the magnetic order is always of the $C$-type and the orbital correlations are of the $G$-type $\underline{\underline{6}}$ On empirical grounds, difference in the ground state of these two compounds is usually attributed to larger lattice distortions in $\mathrm{YVO}_{3} \frac{5}{\underline{5}}$ More specifically, an increase in octahedral tilting in $\mathrm{YVO}_{3}$ supports ferro-type alignment of orbitals along the $c$-axis, hence stabilizing the $G$-type spin order in the ground state of $\mathrm{YVO}_{3}$. This argument has been supported by electronic structure calculations ${ }^{8}$ In vanadates, there are two electrons in the threefold degenerate $t_{2 g}$ level, and it is believed that orbital degrees of freedom are crucial for understanding the interplay between different orderings in these compounds and their highly unusual magnetic properties $.2,3,4,5,6.7$

Based on the above experiments, an effective spin- orbital model has been proposed, ${ }^{9}$ which may properly describe low-energy physics of the cubic vanadates. The $1 \mathrm{D}$ version of this model consists of the $S=1$ Heisenberg spin model with two-fold degenerate orbitals [see Eqs.(1) and (2)]. It has been demonstrated that this effective 1D model contains rich and essential properties inherent in

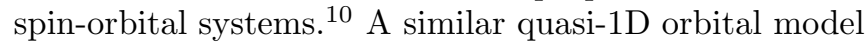
has been also proposed for $\mathrm{LaVO}_{3}, \underline{11}$

In this paper, by calculating the ground-state energy, the spin and orbital correlation functions, and the magnetization curve by means of the density matrix renormalization group (DMRG) method, 12 we investigate quantum phase transitions of the $1 \mathrm{D} S=1$ spin-orbital model, which may be relevant for explaining the magnetic properties of cubic vanadates, in particular, for understanding the phase transitions observed in $\mathrm{YVO}_{3}$. We obtain the phase diagram in the plane of the Hund's coupling $J_{H}$ and the external magnetic field $h$. To make the model more realistic, we introduce also an additional ferromagnetic interaction $V$ to the orbital sector, which may drive the system to an orbital-ferromagnetic phase. We discuss the importance of this interaction for the lowtemperature phase of $\mathrm{YVO}_{3}$ below $77 \mathrm{~K}$.

This paper is organized as follows. After brief discussion of the model in Sec. II, we present in Sec. III the DMRG results for the ground-state energy, the spin/orbital correlation functions in a magnetic field, and show the magnetic phase diagram at absolute zero. In Sec. IV, we address the effects of the ferromagnetic orbital coupling driven by lattice distortions. Brief summary is given in Sec. V.

\section{MODEL}

We start with 1D version of an effective Hamiltonian, which has been proposed to describe the spin and orbital 
properties of cubic vanadates $:^{9,10}$

$$
\begin{aligned}
\mathcal{H} & =J \sum_{i}\left[\frac{1}{2}\left(\mathbf{S}_{i} \cdot \mathbf{S}_{i+1}+1\right) \hat{J}_{i, i+1}+\hat{K}_{i, i+1}\right] \\
& -h \sum_{i} S_{i}^{z},
\end{aligned}
$$

where $\mathbf{S}_{i}$ is the $S=1$ spin operator at the $i$-th site. $J(\equiv$ $\left.4 t^{2} / U\right)$ is the superexchange interaction scale, which we will take as the energy unit in the following discussions. $\hat{J}_{i, j}$ and $\hat{K}_{i, j}$ are the operators acting on the doublydegenerate orbital degrees of freedom, and are given as

$$
\begin{aligned}
& \hat{J}_{i, j}=(1+2 R)\left(\mathbf{T}_{i} \cdot \mathbf{T}_{j}+\frac{1}{4}\right)-r\left(T_{i}^{z} T_{j}^{z}+\frac{1}{4}\right)-R, \\
& \hat{K}_{i, j}=R\left(\mathbf{T}_{i} \cdot \mathbf{T}_{j}+\frac{1}{4}\right)+r\left(T_{i}^{z} T_{j}^{z}+\frac{1}{4}\right),
\end{aligned}
$$

where $\mathbf{T}_{i}$ is the $T=1 / 2$ pseudo-spin operator for the orbital sector. The terms proportional to $R=\eta /(1-3 \eta)$ and $r=\eta /(1+2 \eta)$ with $\eta=J_{H} / U$ originate from the Hund's coupling (normalized by the on-site Coulomb interaction $U$ ). The effective model (11) is derived via the second-order perturbation calculation in $t / U(t$ is the electron hopping amplitude) for the cubic vanadates, where two correlated electrons occupy $t_{2 g}$ orbitals at each site. The microscopic derivation can be found in Ref. 9 . Physically, the external field $h$ in the above Hamiltonian can be regarded as the interchain molecular field present in a realistic $3 \mathrm{D}$ system 13

For $h=\eta=0$, it has been concluded that the ground state of the model (11) is the orbital valence bond (OVB) solid state for which both of the spin and orbital sectors are in a dimer-type singlet state with gapful excitations $\frac{7.10 .14}{}$ Furthermore, it was obtained ${ }^{7.10}$ that a first-order transition from the OVB solid state to the fully polarized spin state with gapless orbital singlet may occur around a critical value $\eta_{c} \sim 0.11$. Since the analysis of Refs. 7,10 is based on the finite-temperature DMRG method, it is difficult to discuss the behavior at lower temperatures. It is thus desirable to study zerotemperature properties to establish the precise phase diagram, which is addressed below in this paper. We further investigate the effects of a magnetic field, as well as of an additional ferromagnetic orbital coupling (which will be specified in Sec. IV).

Before closing this section, we make a brief comment on the spin-orbital model. The $S=1$ model (11) may be regarded as a specific extension of the $S=1 / 2$ spin-orbital model, which has been studied extensively $15,16,17,18,19,20,21$ The ground-state phase diagram of the latter model has been already established by the DMRG method ${ }^{19.20 .21}$ A simple isotropic $S=1$ generalization leads to the model which has the OVB ground state 10.14 We will see below that the extended model, which includes anisotropic orbital interactions, the magnetic field and also an additional ferromagnetic orbital interaction, exhibits interesting quantum phase transitions.

\section{MAGNETIC PHASE DIAGRAM}

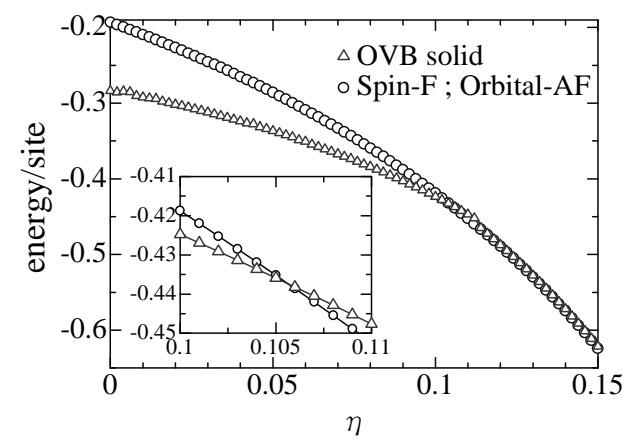

FIG. 1: The energy per site calculated by the infinite DMRG method as a function of the normalized Hund's coupling $\eta$ : the OVB solid state (triangle) and the spin-ferromagnetic state (circle). The inset shows the detail of the $\eta$-dependence in the vicinity of $\eta_{c}$.

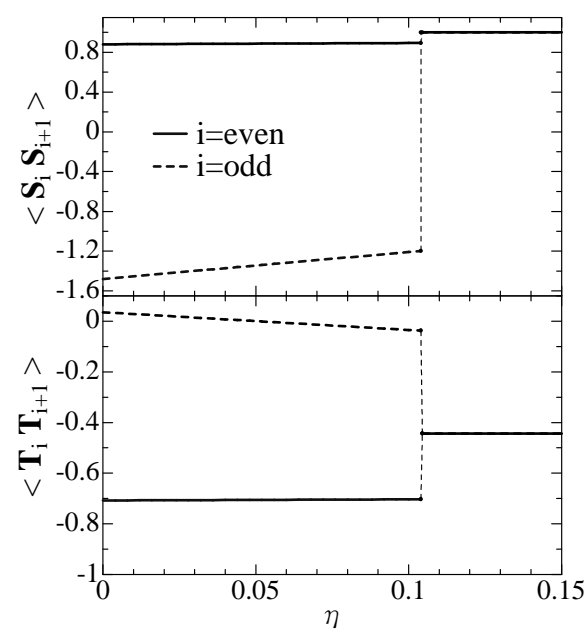

FIG. 2: Spin (upper panel) and orbital (lower panel) correlation functions as a function of the Hund's coupling $\eta$. The site index $i$ is even (solid line) or odd (dashed line). The evenodd $i$-dependence reflects a dimer property of the OVB solid state.

We first consider the effect of the Hund's coupling. To this end, we precisely determine the critical value $\eta_{c}$ between the OVB solid state and the spin-ferromagnetic state. We show the $\eta$-dependence of the energy and the correlation functions calculated by the infinite DMRG $\operatorname{method}^{12}$ for $h=0$ in Figs. 11 and 2 It is seen from Fig. 1 that for small values of the Hund's coupling, the energy of the OVB solid state is lower than that of the spin-ferromagnetic state. In this region, both of the spin and orbital correlation functions show the even-odd $i$-dependence reflecting a dimer character of the OVB 
solid state (see Fig. 2). More precisely, for $i=$ even, two adjacent orbitals form nearly perfect dimer pairs (namely, $\left\langle\mathbf{T}_{i} \cdot \mathbf{T}_{i+1}\right\rangle$ is close to -0.75 ) while the spins prefer the ferromagnetic alignment with a positive value of $\left\langle\mathbf{S}_{i} \cdot \mathbf{S}_{i+1}\right\rangle \simeq 0.9$. On the other hand, for $i=$ odd, the value of the orbital correlation is almost zero while spins form a singlet pair with $\left\langle\mathbf{S}_{i} \cdot \mathbf{S}_{i+1}\right\rangle \simeq-1.5$, which is much less than the value $(=-2)$ expected for a spin-1 isolated dimer ${ }^{22}$ The above behavior is characteristic of the OVB solid state. Increasing $\eta$, the energy of the OVB solid state is getting closer to that of the spinferromagnetic state, and eventually a first-order phase transition occurs between these two phases at a critical value of $\eta_{c}=0.1055 \pm 0.0005$. The detail around the transition is shown in the inset of Fig. 11 Beyond the critical value $\eta_{c}$, the even-odd $i$-dependence of the correlation functions disappears and both of the spin and orbital correlations become spatially uniform with

$$
\left\langle S_{i}^{z} S_{i+1}^{z}\right\rangle=1, \quad\left\langle\mathbf{T}_{i} \cdot \mathbf{T}_{i+1}\right\rangle=-\ln 2+\frac{1}{4},
$$

as should be expected 23 Our conclusion that the ground state changes from the OVB solid to the spinferromagnetic state, and also the value of $\eta_{c}$ at which the transition occurs, is in good agreement with Ref. 10.

The effective spin (orbital) exchange coupling constants, which are determined by local orbital (spin) correlations, change drastically at the phase transition. Just below $\eta_{c}$, the spin interactions $J_{s}=\left\langle\hat{J}_{i, i+1}\right\rangle / 2$ are equal to $J_{F} \simeq-0.38$ within the ferromagnetic bonds, and $J_{A F} \simeq 0.05$ between the spins belonging to different dimers. Above the transition, $J_{s} \simeq-0.21$ in all the bonds, supporting uniform Heisenberg-like orbital dynamics. Similarly, the orbital exchange constant $J_{\text {orb }}=$ $(1+2 R)\left\langle\mathbf{S}_{i} \cdot \mathbf{S}_{i+1}+1\right\rangle / 2+R$ changes from: $J_{\text {orb }} \simeq 1.4$ $(\simeq 0.02)$ in strong (weak) bonds below $\eta_{c}$, to the uniform value $J_{\text {orb }} \simeq 1.46$ above $\eta_{c}$.

Let us next consider the magnetic properties of the model (11) for finite values of $h$. We have calculated the magnetization curve as well as the correlation functions. The results of the magnetization are shown in Fig. 3 for two typical choices of the Hund's coupling. As seen from the case of $\eta=0$ in Fig. 3] the ground state in small fields is still in the OVB solid state, rendering the magnetization zero up to a critical field $h_{c 1}$ due to the existence of the spin gap. Beyond the critical field $h_{c 1}$, the spin sector becomes gapless, resulting in the square-root increase of the magnetization near the critical field $h_{c 1}$. We note that this transition in the spin sector is a kind of Pokrovsky-Talapov transition, i.e. an insulator to gapless-liquid transition triggered by fillingcontrol $^{24}$ Therefore, the ferro/antiferromagnetic alternating spin correlation still exists even in the gapless phase. The magnetization then smoothly increases until we encounter a sudden jump at the second critical field $h_{c 2}$, where the system undergoes a first-order transition to the fully polarized spin state with the orbital sector forming a gapless $T=1 / 2$ Heisenberg pseudo-spin chain. Note that in contrast to the spin sector, the orbital sector is always gapful for $0 \leq h<h_{c 2}$. For $\eta=0$ $(\eta=0.05)$, the corresponding critical field is $h_{c 2}=0.122$ $\left(h_{c 2}=0.065\right)$.

Shown in Fig. 4 are the correlation functions for $\eta=0$ under magnetic fields. As mentioned above, in weak fields, both of spin and orbital correlations exhibit the even-odd dependence characteristic of the OVB solid state. Their values are unchanged up to $h_{c 1}$, beyond which the even-odd dependence is gradually suppressed. Notice that the spin correlation function shows a cusp singularity at $h_{c 1}$ while the orbital correlation function smoothly changes. This is because the spin sector shows an insulator to gapless-liquid transition, whereas the orbital sector still stays in the gapful phase, which is affected by magnetic fields indirectly via the spin-orbital interaction. In magnetic fields beyond $h_{c 2}$, the spin and orbital correlation functions take the values given in (3) corresponding to the fully polarized spin state with a gapless orbital Heisenberg chain. Similar behavior in the field dependence of the correlation functions is observed for other choices of the Hund's coupling, as far as the ground state is in the OVB solid state at $h=0$.

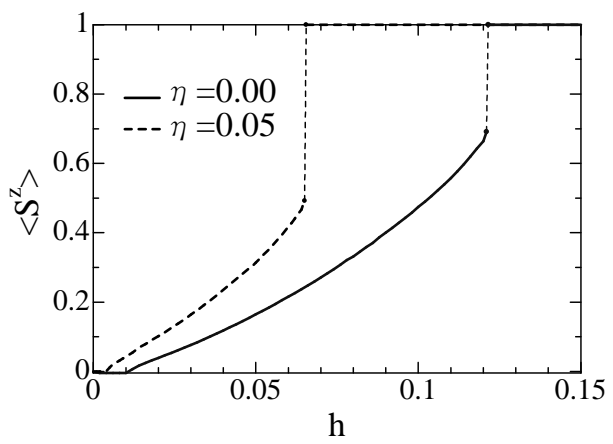

FIG. 3: Magnetization curves for $\eta=0$ (solid line) and $\eta=0.05$ (dashed line). The magnetization becomes finite for $h>h_{c 1}$ and exhibits a jump at $h_{c 2}$ characteristic of the first-order phase transition.

We thus end up with the zero-temperature magnetic phase diagram, which is shown in Fig. [5 There are three distinct phases in the $\eta$ - $h$ plane. In the region of weak fields and weak Hund's couplings, the OVB solid state is stabilized in the phases I and II; the orbital sector is gapful while the spin sector is gapful (gapless) in the phase I (II). The transition from the phase I to II is of Pokrovsky-Talapov type. On the other hand, the transition from the phase II to the spin-ferromagnetic phase III is of first order.

For the spin/orbital dimerized state at $\eta=0$, we estimated the spin gap from the magnetization curve and obtained $\Delta_{s} \sim 0.01 J$. This is actually much larger than the Haldane gap that one would expect from a simple picture of spin- 2 dimers coupled by a weak antiferromagnetic exchange. Indeed, at $\eta=0$ an effective spin 


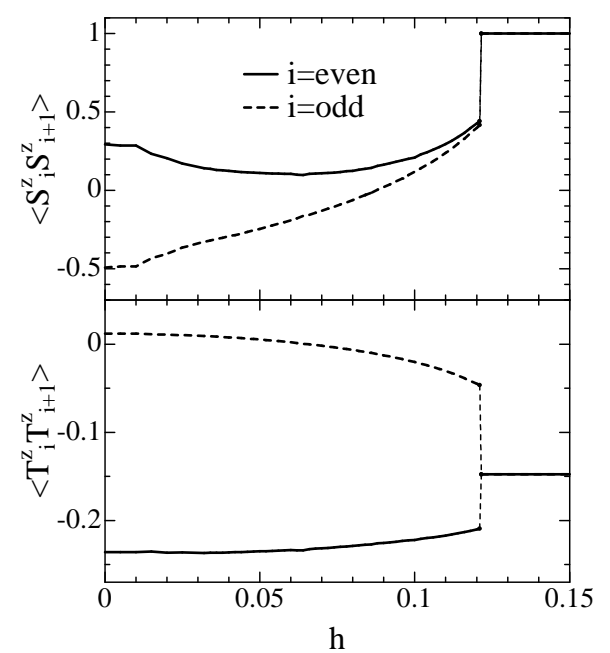

FIG. 4: Spin (upper panel) and orbital (lower panel) correlation functions for $\eta=0$ in a magnetic field. The site index $i$ is even (solid line) or odd (dashed line).

exchange constant between the dimers is estimated as $J_{\text {eff }}=\frac{1}{8}\left\langle\mathbf{T}_{i} \cdot \mathbf{T}_{j}+\frac{1}{4}\right\rangle \simeq 0.036$. This would give the spin- 2 Haldane gap about $0.085 J_{\text {eff }} \simeq 0.003$ only ${ }^{25.26}$ The large spin gap in the spin-orbital model is due to a dynamical coupling between two sectors, as discussed in Ref. 10. We notice that our result for the spin gap is still much smaller than $\Delta_{s} \sim 0.041 \mathrm{~J}$, obtained in Ref. 10 from the finite-temperature spin susceptibility $\chi$ computed by the transfer-matrix DMRG method. As the latter method is available at finite temperatures only, Ref. 10 estimates the spin gap by extrapolating the data with a function of $\chi \sim \exp \left(-\Delta_{s} / T\right) / \sqrt{T}$. We think that the above discrepancy may arise from this (somewhat ambiguous) extrapolation procedure.

\section{EFFECTS OF THE FERROMAGNETIC ORBITAL INTERACTION}

For representative values of $J_{H} \simeq 0.68 \mathrm{eV}$ and $U-$ $\frac{20}{9} J_{H} \simeq 4.5 \mathrm{eV}$ for vanadates,${ }^{27}$ one obtains that the ratio $\eta=J_{H} / U \simeq 0.11$ is slightly above its critical value. Therefore, our results are consistent with the experimental fact that the ferromagnetic chain is stabilized along the $c$-axis in the ground state of $\mathrm{LaVO}_{3} \stackrel{6}{ }$ However, the superexchange model (11) alone is not sufficient to understand the origin of the spin- $G$ type ground state in $\mathrm{YVO}_{3}$, and also to understand the physics behind the spin/orbital reordering at $T=77 \mathrm{~K}$. In order to describe low-temperature properties of $\mathrm{YVO}_{3}$ more precisely, it is desirable to take into account the pure-orbital interaction in addition to the spin/orbital couplings involved in the Hamiltonian (11). The orbital interaction to be included

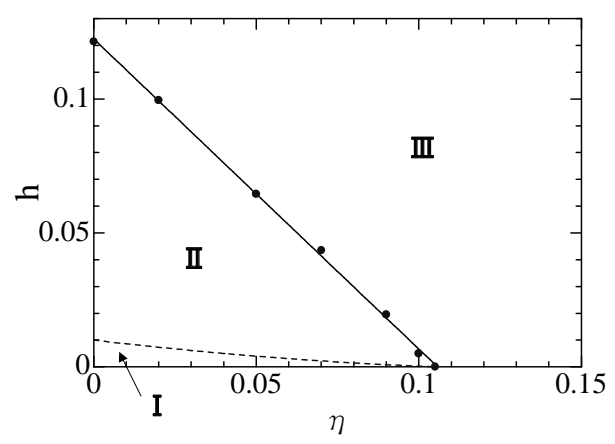

FIG. 5: Magnetic phase diagram: Phase I represents the OVB solid state with zero magnetization (spin-dimer state). Phase II is also in the OVB solid state, but with a finite magnetization $0 \leq\left\langle S_{i}^{z}\right\rangle \leq 1$. Phase III is the spin-ferromagnetic phase with a gapless $T=1 / 2$ orbital chain. The first-order phase transition occurs at the boundary denoted by the solid line.

has the following Ising-like form,,$\frac{9}{}$

$$
\mathcal{H}_{o}=-V \sum_{i} T_{i}^{z} T_{i+1}^{z}
$$

This describes a ferromagnetic orbital coupling, which competes with the antiferromagnetic orbital coupling in the Hamiltonian (11). Physically, this term is important because apart from the superexchange mechanism there is also a lattice contribution to the orbital interactions. As mentioned in the introduction, tilting of octahedra in perovskites (caused by ionic mismatch effects) leads to a tendency that orbitals are aligned ferromagnetically along the $c$-axis. This effect is stronger when we go from La- to Y-based compound 8 We thus expect that the ferromagnetic Ising orbital coupling along the $c$-axis may be particularly relevant for $\mathrm{YVO}_{3}$, in order to explain why its ground state shows spin-antiferro/orbitalferromagnetic structure along the $c$-axis.

We choose here three different values of the Hund's coupling and calculate the energy as a function of $\mathrm{V}$. In Fig. [6(a), the $V$-dependence of the energy for three competing states is shown for the OVB ground state $(\eta=0.05)$. There is a direct first-order transition from the OVB state to the orbital-ferromagnetic state. On the other hand, when the Hund's coupling is slightly above the critical value $\eta_{c}(\eta=0.11)$, we encounter different behavior, as seen in Fig. 6(b). Namely, the ground state is the spin-ferromagnetic (orbital-ferromagnetic) state for small $V$ (large $V$ ), but for intermediate $V$, the OVB solid state is stabilized, which results from the competition of the above two (spin-ferromagnetic or orbital ferromagnetic) interactions. Stabilization of the orbital disordered OVB state by finite $V$-interaction (induced by lattice distortion) is a remarkable result. The physics behind this observation is that the lattice-driven interaction (4) introduces frustration into the orbital sec- 
tor, competing with antiferro-type alignment of orbitals due to the superexchange process. For even larger $\eta$ [Fig. 6[(c)], $V$ induces a direct phase transition from the spin-ferromagnetic state to the orbital-ferromagnetic state. (a) $\eta=0.05$

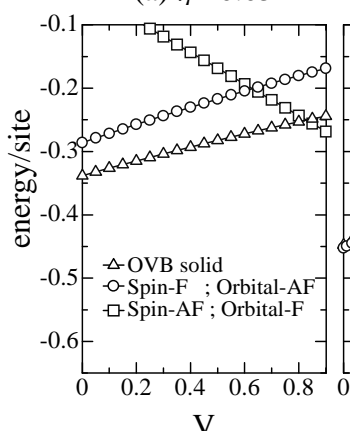

(b) $\eta=0.11$

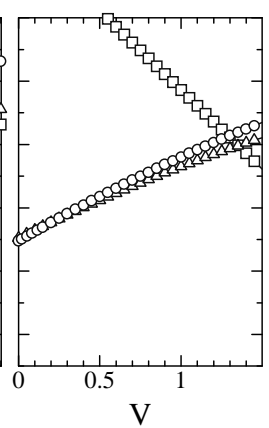

(c) $\eta=0.15$

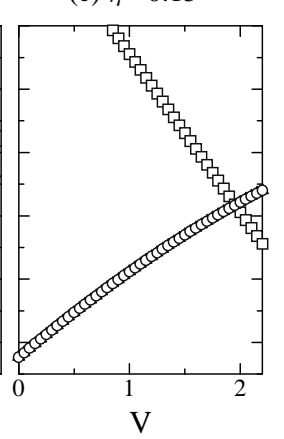

FIG. 6: The energy per site as a function of the orbital coupling $V$ for several choices of $\eta$; the OVB solid state (triangle), the spin-ferromagnetic state (circle), orbital-ferromagnetic state (square). Notice that the first-order transition occurs once for (a) and (c), while it occurs twice for (b) when the value of $V$ is increased.

The phase diagram thus obtained in the $\eta$ - $\mathrm{V}$ plane is shown in Fig. 7 There are three distinct phases in this figure. For small $\eta$ and $V$, the OVB solid state is stabilized in the phase I, which is driven to the orbitalferromagnetic phase $\mathrm{IV}$ with the increase of $V$. In the latter phase $\mathrm{I}$, the spin sector is described by an $S=1$ isotropic antiferromagnetic spin chain, and thus its ground state is a spin liquid with Haldane-gap excitations. In the spin-ferromagnetic phase III, the orbital sector is described by the $X X Z$ pseudo-spin model, which results from (11) and (41):

$$
\mathcal{H}=\widetilde{J} \sum_{i} \mathbf{T}_{i} \cdot \mathbf{T}_{i+1}-V \sum_{i} T_{i}^{z} T_{i+1}^{z} .
$$

Here $\widetilde{J}=J /(1-3 \eta)$, and we have discarded irrelevant constant terms. According to the well known results for the $S=1 / 2 X X Z$ spin chain with ferromagnetic Ising anisotropy ${ }^{28}$ the ground state is in a gapless liquid phase for $V<2 \widetilde{J}$, and in a fully polarized ferromagnetic phase for $V \geq 2 \widetilde{J}$. Therefore it might be possible to have the orbital-ferro/spin-ferromagnetic ground state in the region $V \geq 2 /(1-3 \eta)$ in units of $J$. However, it turns out that since the first-order transition from the phase III to IV occurs at the phase boundary shown in Fig. 7 there is no available window of the parameters which can stabilize the orbital-ferro/spin-ferromagnetic ground state.

The ferromagnetic orbital interaction $V$ may be relevant to the first-order phase transition observed in $\mathrm{YVO}_{3}$ at $T=77 \mathrm{~K}$, where the spin ordering changes from the $G$-type (below $77 K$ ) to the $C$-type (above $77 \mathrm{~K})$ structure. If we focus on the pattern of ordering in the $c$-direction, the transition occurs from the spin-antiferro/orbital-ferromagnetic ground state to the spin-ferro/orbital-antiferromagnetic state. Furthermore, recent neutron scattering experiments have revealed a substantial modulation of the ferromagnetic spin couplings along the $c$-direction at $T>77 \mathrm{~K}$, which indicates the appearance of orbital dimer correlations at finite temperatures ${ }^{\underline{7}}$ According to the results obtained here, such a temperature-driven first-order transition may possibly occur when the system is located in the phase $\mathrm{IV}$ near the tricritical point in the phase diagram (Fig. (7), and thus close to the phases III and I. For example, $V \simeq 1.5$ may be a reasonable choice for $\eta \simeq 0.12$, which may give rise to the competition of the phases at finite temperatures. This is in fact quite realistic situation, as the atomic value of Hund's coupling parameter for vanadates is indeed close to this value ${ }^{27}$ Finally, we wish to mention that the gapless orbital-liquid (gapful spinliquid) state in the phase III (IV) is expected to show an orbital (spin) antiferromagnetic order when realistic three-dimensional effects are properly taken into account, being consistent with experimental findings.

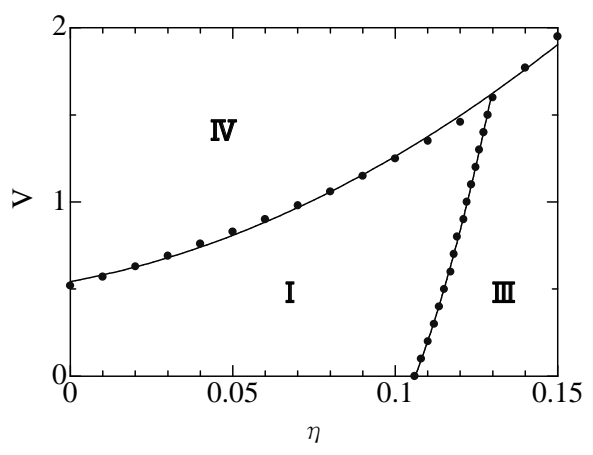

FIG. 7: Phase diagram in the $\eta$-V plane; Phase $\mathrm{I}$ is the OVB phase, III is the spin-ferromagnetic phase, and IV is the orbitalferromagnetic phase stabilized by the orbital ferromagnetic Ising term. All the phase transitions are of first-order. Note that the orbital (spin) sector in the phase III (IV) is a gaplessorbital (gapful-spin) liquid.

\section{SUMMARY}

We have investigated quantum phase transitions of the 1D $S=1$ spin-orbital model relevant to cubic vanadates. By means of the DMRG calculation, we have determined the zero-temperature phase diagram in a magnetic field. We have found that the magnetic field induces the phase transitions twice for the case of OVB ground state. The lower-field transition is of Pokrovsky-Talapov type, which drives the spin sector to a gapless spin liquid while keeping the orbital sector always gapful. On the other hand, the high-field transition is first order to the spin-ferromagnetic state, where the orbital state is driven to a gapless isotropic $T=1 / 2$ orbital chain. 
We have considered the evolution of spin and orbital correlations as a function of the Hund's coupling, and obtained that the ground state of the superexchange model is spin-ferromagnetic for the realistic values of $J_{H}$. This is consistent with the formation of ferromagnetic spin chains along the $c$-axis in $\mathrm{LaVO}_{3}$.

The introduction of the ferromagnetic Ising coupling $V$ to the orbital sector induces a first-order phase transition to the orbital-ferro/spin-antiferromagnetic state. This may explain the stabilization of the $G$-type spin order observed in $\mathrm{YVO}_{3}$ at temperatures below $77 \mathrm{~K}$. We found also that the intermediate values of $V$-interaction may stabilize the spin/orbital dimer phase. This phenomenon reflects competition between the superexchange and lattice effects, and, surprisingly enough, it occurs for $J_{H}$-values realistic for vanadates. The proximity of different spin/orbital states in the ground state may result in an entropy-driven phase transition at finite temperature, similar to that observed in $\mathrm{YVO}_{3}$. In the future study, it is desirable to discuss whether such a temperature-driven first-order transition may be indeed possible in the spinorbital model including the ferromagnetic orbital interaction and also the interchain coupling effects. This may provide a key to clarify some essential spin/orbital properties of $\mathrm{YVO}_{3}$ at low temperatures.

\section{Acknowledgements}

This work was partly supported by a Grant-in-Aid from the Ministry of Education, Science, Sports and Culture of Japan. A part of computations was done at the Supercomputer Center at the Institute for Solid State Physics, University of Tokyo and Yukawa Institute Computer Facility.
* Present address: NTT Basic Research Laboratories, Atsugi, Kanagawa 243-0198, Japan.

${ }^{1}$ H. Kawano, H. Yoshizawa and Y. Ueda, J. Phys. Soc. Jpn. 63, 2857 (1994).

2 Y. Ren, T. T. M. Palstra, D. I. Khomskii, E. Pellegrin, A. A. Nugroho, A. A. Menovsky, and G. A. Sawatzky, Nature 396, 441 (1998).

${ }^{3}$ Y. Ren, T. T. M. Palstra, D. I. Khomskii, A. A. Nugroho, A. A. Menovsky, and G. A. Sawatzky, Phys. Rev. B 62, 6577 (2000).

${ }^{4}$ M. Noguchi, A. Nakazawa, S. Oka, T. Arima, Y. Wakabayashi, H. Nakao, and Y. Murakami, Phys. Rev. B 62, R95271 (2000).

${ }^{5}$ G. R. Blake, T. T. M. Palstra, Y. Ren, A. A. Nugroho, and A. A. Menovsky, Phys. Phys. Lett. 87, 245501 (2002).

6 S. Miyasaka, Y. Okimoto, and Y. Tokura, J. Phys. Soc. Jpn. 71, 2086 (2002).

7 C. Ulrich, G. Khaliullin, J. Sirker, M. Reehuis, M. Ohl, S. Miyasaka, Y. Tokura, and B. Keimer, cond-mat/0211589

8 T. Mizokawa, D. I. Khomskii, and G. A. Sawatzky, Phys. Rev. B 60, 7309 (1999).

9 G. Khaliullin, P. Horsch, and A. M. Oleś, Phys. Phys. Lett. 86, 3879 (2001).

10 J. Sirker and G. Khaliullin, Phys. Rev. B 67, 100408 (2003).

11 Y. Motome, H. Seo, Z. Fang, and N. Nagaosa, Phys. Phys. Lett. 90, 146602 (2003).

12 S. R. White, Phys. Rev. Lett. 69, (1992) 2863; Phys. Rev. B. 48, (1993) 10345.

13 We do dot consider here the interchain interactions in orbital sector. As discussed in Refs. 9 11, orbital interactions in the spin C-phase are essentially of $1 \mathrm{D}$ nature.

14 S.-Q. Shen, X. C. Xie, and F. C. Zhang, Phys. Phys. Lett.
88, 027201 (2002).

15 B. Sutherland, Phys. Rev. B 12, 3795 (1975).

16 I. Affleck, Nucl. Phys. B 265, 409 (1986).

17 T. Itakura and N. Kawakami, J. Phys. Soc. Jpn. 64, 2321 (1995).

18 P. Azaria, A. O. Gogolin, P. Lecheminant, and A. A. Nersesyan, Phys. Rev. Lett. 83, 624 (1999); P. Azaria, E. Boulat, and P. Lecheminant, Phys. Rev. B 61, 12112 (2000).

19 C. Itoi, S. Qin, and I. Affleck, Phys. Rev. B. 61, 6747 (2000).

20 S. K. Pati, R. R. P. Singh, and D. I. Khomskii, Phys. Rev. Lett. 81, 5406 (1998).

21 Y. Yamashita, N. Shibata, and K. Ueda, J. Phys. Soc. Jpn. 69, 242 (2000); Physica B 281-282, 542 (2000).

22 The value $\left\langle\mathbf{S}_{i} \cdot \mathbf{S}_{i+1}\right\rangle \simeq-1.5$ for $i=$ odd bonds follows in fact from the following simple consideration: Two adjacent dimers (each with spin $S_{D}=2$ ) do couple antiferromagnetically, thus the total spin of two dimers is zero. In this state, $\left\langle\mathbf{S}_{i} \cdot \mathbf{S}_{i+1}\right\rangle \simeq-\frac{1}{4} S_{D}\left(S_{D}+1\right)=-1.5$.

${ }^{23}$ H. Bethe, Z. Phys. 71, 205 (1931); L. Hulthen, Arkiv. Math. Astro. Fys. 26A, 938 (1938).

24 V. L. Pokrovsky and A. L. Talapov, Phys. Rev. Lett. 42, 65 (1979); Zh. Eksp. Teor. Fiz. 78, 269 (1980) [Sov. Phys. JETP 51, 134 (1980)].

25 S. Yamamoto, Phys. Rev. Lett. 75, 3348 (1995).

26 U. Schollwöck and T. Jolicoeur, Europhys. Lett. 30, 493 (1995).

27 T. Mizokawa and A. Fujimori, Phys. Rev. B 54, 5363 (1996).

28 J. des Cloizeaux and M. Gaudin, J. Math. Phys. 7, 1384 (1966). 